\title{
An investigation of semiconductor nanoparticles for application to all-optical switching
}

\author{
Brandon Born, Simon Geoffroy-Gagnon, and Jonathan F. Holzman \\ Integrated Optics Laboratory, The University of British Columbia, School of Engineering \\ 3333 University Way, Kelowna, BC, Canada, V1V 1V7
}

\begin{abstract}
A practical all-optical switch is necessary to alleviate electronic bottlenecks in fibre optic networks. Thus, a new alloptical switch is introduced here-exhibiting femtojoule switching energies and femtosecond switching times. The alloptical switches use $40 \mu \mathrm{m}$ dielectric spheres to direct high-intensity photonic nanojets into peripheral coatings of semiconductor nanoparticles. Semiconductor nanoparticle coatings of $\mathrm{Si}, \mathrm{CdTe}, \mathrm{InP}$, and $\mathrm{CuO}$ are studied and found to yield switching energies of approximately $1 \mathrm{pJ}, 500 \mathrm{fJ}, 400 \mathrm{fJ}$, and $300 \mathrm{fJ}$ with switching times of $2 \mathrm{ps}, 2.3 \mathrm{ps}, 900 \mathrm{fs}$, and $350 \mathrm{fs}$, respectively.
\end{abstract}

Keywords: Nanophotonics, nanoparticles, all-optical switching, photonic nanojet, pump-probe, ultrafast.

\section{INTRODUCTION}

All-optical switching has been the focus of a great deal of research in recent years. The research has been spurred by the potential for all-optical switching to support terabit-per-second processing with protocol transparency in all-optical networks. But the implementation of effective all-optical networks has remained elusive to a large extent, given the unanswered challenges for the basic element in these systems - the all-optical switch (AOS).

The AOS is the basic element that facilitates all-optical modulation. The AOS utilizes a control beam to induce modulation on a coincident signal beam, and the performance of such a process is defined by two key metrics. The first metric pertains to the switching energy. The switching energy quantifies the ease with which the control beam modulates the coincident signal beam, and it is quoted in terms of the control beam's pulse energy. It is desirable to have this switching energy be as low as possible to facilitate low-power operation in complex all-optical networks. Femtojoule switching energies are the goal ${ }^{[1]}$. The second metric pertains to the switching time. The switching time quantifies the duration of the control-signal beam interaction, following impulsive excitation by the control beam. For obvious reasons, the switching time should be as short as possible to facilitate ultra-broadband all-optical switching. Femtosecond switching times are the goal ${ }^{[2]}$.

Numerous AOS architectures have emerged in the literature for the implementation of all-optical switching with femtojoule switching energies or femtosecond switching times. The AOS architectures have taken the form of quantum $\operatorname{dots}^{[3]}$, ring resonators ${ }^{[4]}$, photonic crystal resonators ${ }^{[5]}$, and many others. Unfortunately, the simultaneous realization of femtojoule switching energies and femtosecond switching times has proven to be far more difficult. The demonstrated AOS architectures often take the form of resonators, to facilitate energy storage and operate with low switching energies, but the resulting resonance introduces cavity lifetimes, which increase the switching times. Thus, for resonance-based AOS architectures the goals of femtojoule switching energies and femtosecond switching are mutually exclusive ${ }^{[6],[7]}$.

In this work, we explore the process of all-optical switching through an alternative AOS architecture. The AOS architecture is implemented as a dielectric microsphere with semiconductor nanoparticles embedded on its surface ${ }^{[8]}$. It is shown that the characteristics of the dielectric microsphere, being the refractive index, $n$, and the diameter, $d$, can be tailored to bring about high-intensity focusing at the rear surface of the dielectric microsphere-in the form of a photonic nanojet ${ }^{[9],[10],[11]}$. The characteristics of the dielectric microsphere are optimized in an effort to maximize the photonic nanojet's intensity and enhance the all-optical modulation between the co-propagating control and signal beams. Such a process leads to operation with low switching energies. It is also shown that the characteristics of the semiconductor nanoparticles, being the semiconductor composition and the radius, $a$, can be tailored to have the co-propagating control and signal beams exhibit strong nonlinear interactions with ultrashort switching times. A select group of semiconductor nanoparticle types and radii are investigated in this work to establish operation with ultrashort switching times. It is shown that the fully-optimized AOS architecture can (concurrently) achieve the goals of femtojoule switching energies and femtosecond switching times.

Ultrafast Phenomena and Nanophotonics XX, edited by Markus Betz, Abdulhakem Y. Elezzabi, Proc. of SPIE Vol. 9746, 97460E · C 2016 SPIE · CCC code: 0277-786X/16/\$18

doi: $10.1117 / 12.2208638$

Proc. of SPIE Vol. $974697460 \mathrm{E}-1$ 


\section{THE PHOTONIC NANOJET}

A superlens can create a high-intensity subdiffractional focal spot. In this study, a superlens, in the form of a dielectric microsphere, is used to focus a beam into a subdiffractional nonevanescent focal spot, referred to as a photonic nanoje ${ }^{[9],[10]}$. The photonic nanojet forms when a beam that is incident on the front surface of a suitably designed microsphere is refracted and focused on the rear surface of the microsphere. Especially high intensities can be achieved, which can be exploited for reducing the switching energy required for interaction between the control and signal beams.

A dielectric sphere's properties must be optimized to achieve the desired focusing, as the formation of the photonic nanojet relies on the microsphere's diameter, $d$, and refractive index, $n$. Large spheres, made with contemporary glasses, conform to the thick lens formula, and it can be shown ${ }^{[12]}$ that the highest intensity on the rear surface of the sphere is realized with a refractive index of $n \approx 2$. Small spheres, that have sizes comparable to the wavelength, require threedimensional electromagnetic analysis. In this case, the photonic nanojet is best modeled by the Lorenz-Mie Theory ${ }^{[11]}$, which derives a harmonic solution in spherical coordinates. Microspheres with varying diameters, $d \leq 30 \mu \mathrm{m}$, and refractive indices, $1.5 \leq n \leq 2.0$, are simulated with Lorenz-Mie Theory, and the intensities at each microsphere's rear surface are recorded. The results are shown in Figure 1 with each pixel representing this recorded intensity from over one hundred thousand individual Lorenz-Mie Theory simulations. It is apparent that the highest intensities of the photonic nanojets trend from lower refractive indices, starting at $n \approx 1.75$, to higher refractive indices, as illustrated by the red trendline. The trendline approaches the thick lens formula solution of $n \approx 2$ for sufficiently large diameters.

The interferometric fringes in Figure 1 are indicative of resonance and are ideally avoided for the purposes of this study on all-optical switching. The fringes result from i. longitudinal modes, which form along the optical axis and lead to the steep high-spatial-frequency fringes seen in the intensity map, and ii. coupled longitudinal-whispering-gallery modes ${ }^{[13]}$, which form interferometric beating and lead to the sloped low-spatial-frequency fringes. This resonance can be eliminated by using structures that are sufficiently large, compared to the wavelength, or pulse durations that are less than the round-trip cavity time. This study implements the photonic nanojet with a microsphere having a diameter of $d=40 \mu \mathrm{m}$ and a refractive index of N-LASF9 glass, $n=1.83$ (the combination of which yield a maximized intensity).

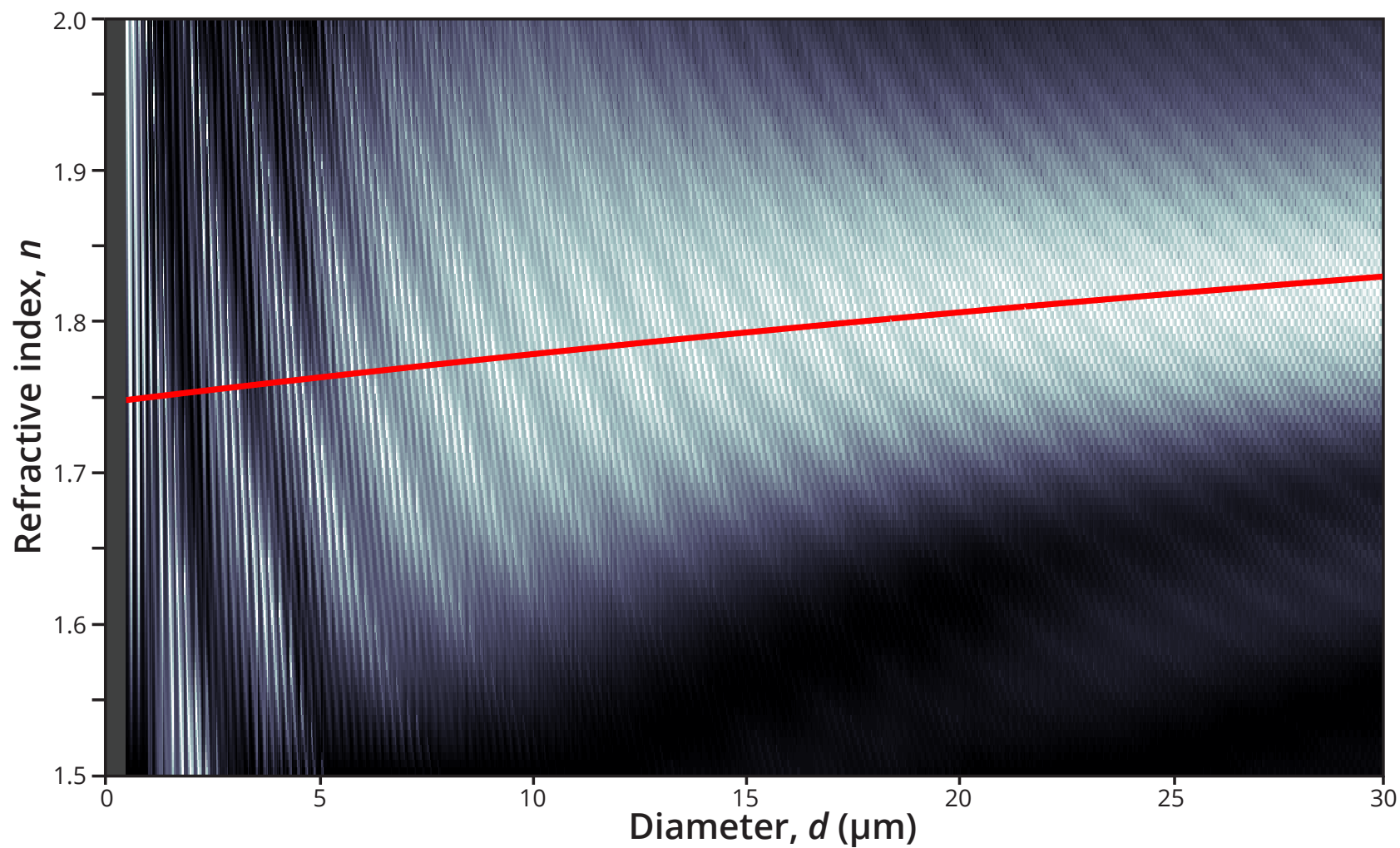

Figure 1. Photonic nanojet maximum intensities. Theoretical nanojet intensities, for a $\lambda=780 \mathrm{~nm}$ beam, at the rear surface of the sphere versus the refractive index, $n$, and diameter, $d$, of the sphere. Lorenz-Mie Theory is used to generate the intensity distribution from over a hundred thousand individual simulations. The intensities of the greyscale intensity map are shown in white, with the results normalized for all $n$ with constant $d$. The trendline for the maximum intensities is shown as a red curve. 


\section{NANOPARTICLE FREE-CARRIER DYNAMICS}

The semiconductor nanoparticles are investigated here for implementation with the photonic nanojet for all-optical switching. Both the semiconductor composition and nanoparticle size are considered.

In this application, the semiconductor composition must be chosen to establish a high nonlinearity in the presence of the control beam to affect the signal beam. A sufficiently strong interaction must occur over the short propagation length of the photonic nanojet, with only femtojoule switching energies. The best option to achieve this is with third-order Kerr nonlinearities having resonance with the semiconductor's bandgap. This is applied here with a control beam wavelength of $775 \mathrm{~nm}$ and a signal beam wavelength of $1550 \mathrm{~nm}$, such that the above-bandgap control beam induces free-carriers to alter the transmission of the below-bandgap signal beam. Typically, third-order effects that are resonant will have higher nonlinear coefficients than those of nonresonant configurations. Also nonresonant configurations would be ill-suited for operation with the photonic nanojet as such configurations typically require phase-matched beams over millimetre or centimetre lengths ${ }^{[14]}$. This study proceeds using resonant third-order nonlinearity, however, there is an additional constraint; the free-carriers must recombine on a femtosecond timescale to enable terabit-per-second processing.

The size of the nanoparticles greatly impacts the free-carrier lifetime due to the effects of surface recombination. Surface states promote free-carrier recombination in proportion to the surface area. Prior research has shown all-optical switching with cylindrical ${ }^{[15]}$ and spherical ${ }^{[16]}$ semiconductor nanostructures with large surface state densities can speed up recombination. This study uses spherical nanoparticles, having the higher surface-area-to-volume ratio of the two.

The effects of the control beam on the signal beam are described in this section, and some guiding design principles are revealed. The induced free-carriers by the control beam can affect the signal beam through both free-carrier dispersion and free-carrier absorption, as well as through possible state-filling. Drude Theory states that the free-carrier density, $N(t)$, will decrease the semiconductor's refractive index (free-carrier dispersion) and increase the bulk absorption (free-carrier absorption). This in turn will create respective increasing and decreasing changes in signal beam's transmission. However, for nanostructures, bulk absorption becomes negligible as surface changes dominate ${ }^{[17]}$. The outcome is a positive change in transmission from free-carrier dispersion. There is also the possibility of state-filling effects as a result of mid-gap defect states introduced into the semiconductor lattice by the nanostructure. This allows the signal beam to populate mid-gap surface states with free-carriers, which is seen as absorption for the signal beam. With the introduction of the control beam, however, the signal beam absorption is diminished due to the excitation of freecarriers by the control beam ${ }^{[18],[19]}$. It can be difficult to discern the contributions of free-carrier absorption from statefilling when measuring the differential transmission of the signal beam, $\Delta T(t) / T$. However, it can be safely assumed that both contributions are proportional to the free-carrier density, $N(t)^{[20]}$.

The free-carrier density is modelled as a spherically symmetric distribution, $N(r, t)$, varying radially, $r$, and with time, $t$. The nanoparticle begins with the initially uniform free-carrier density of $N_{0}$, based on the assumption of uniform illumination by the control beam. Then the free-carriers evolve according to

$$
\frac{\partial N(r, t)}{\partial t}=N_{0} \delta(t)+D \nabla^{2} N(r, t)-\frac{N(r, t)}{\tau_{\mathrm{b}}},
$$

with the delta function, $\delta(t)$, defining the photogeneration of free-carriers at $t=0$, the diffusion coefficient, $D$, defining the transport of free-carriers, and the bulk recombination lifetime, $\tau_{\mathrm{b}}$, defining the semiconductor's inherent bulk recombination rate. Free-carrier transport continues outward, from the middle of the sphere outward to the radius, $a$. At the radius, the free-carriers undergo surface recombination according to the boundary condition $-D \partial N(r=a, t) / \partial r=$ $S_{\mathrm{v}} N(r=a, t)$. Here, the rate of surface recombination is defined by the surface recombination velocity is $S_{\mathrm{v}}$. The solution of Eq. (1) and this boundary condition yields the free-carrier density distribution, $N(r, t)$, and this distribution is averaged in space to quantify a transient free-carrier density, $N(t)$. This transient free carrier density is used to quantify the differential transmission of the signal beam, $\Delta T(t) / T$.

Direct solutions of Eq. (1) and its boundary condition can be acquired by a frequency-domain (Laplace) approach. For such an approach, the solution is assumed to be independent of the polar angle, $\theta$, and the Laplace solution, $N(r, s)$, is created. The inverse Laplace transform is then computed by way of

$$
N(r, t)=\mathcal{L}^{-1}\{N(r, s)\}=\mathcal{L}^{-1}\left\{\frac{N_{0}}{s+1 / \tau_{\mathrm{b}}}\left[1-\frac{a}{r} \frac{S_{\mathrm{v}} \sinh (k r)}{\left(S_{\mathrm{v}}-D / a\right) \sinh (k a)+D k \cosh (k a)}\right]\right\},
$$

where $k=\sqrt{s / D+1 / \tau_{\mathrm{b}}}$. The inverse Laplace transform, $N(r, t)$, is computed numerically for the desired values of the parameters $a, D$, and $S_{\mathrm{v}}$. 
For a deeper understanding of the underlying free-carrier dynamics in the nanoparticle, solutions to Eq. (1) and its boundary condition can be acquired by a time-domain approach. To do this, a general solution is generated for Eq. (1). The general solution is a function of the radial dimension, $r$, polar angle, $\theta$, and time, $t$, and it is defined by ${ }^{[21]}$

$$
N(r, \theta, t)=\sum_{m=0}^{\infty} \sum_{i=1}^{\infty} \Gamma_{m, i} j_{m}\left(\lambda_{m, i} r / a\right) P_{m}(\cos \theta) \mathrm{e}^{-t / \tau_{m, i}},
$$

where $j_{m}(\cdot)$ are the spherical Bessel functions of the first kind and $P_{m}(\cdot)$ are the Legendre polynomials. Both of these functions are of order $m$. Here, the eigenvalues are defined by $\lambda_{m, i}$, the decay coefficients are defined by $\Gamma_{m, i}$, and the time constants are defined by $\tau_{m, i}$. The boundary condition at the radius is used to create the characteristic equation of $j_{m}\left(\lambda_{m, i}\right) / j_{m}^{\prime}\left(\lambda_{m, i}\right)=-D \lambda_{m, i} /\left(S_{\mathrm{v}} a\right)$, and this characteristic equation is used to generate the discrete eigenvalues, $\lambda_{m, i}$. An initial uniform free-carrier density, $N_{0}$, is applied to generate the decay coefficients, $\Gamma_{m, i}$. The desired time constants, $\tau_{m, i}$, can then be generated by

$$
\frac{1}{\tau_{m, i}}=\frac{1}{\tau_{\mathrm{b}}}+\frac{D \lambda_{m, i}^{2}}{a^{2}}
$$

This study assumes $\theta$ independence for the solution of $N(r, \theta, t)$, which yields a finite term for only $m=0$ on the first summation. In addition, a first-order approximation is applied to keep only the $i=1$ term for the second summation. Thus, Eq. (3) can then be simplified to be

$$
N(r, t) \approx \Gamma \frac{\sin (\lambda r / a)}{\lambda r / a} \mathrm{e}^{-t / \tau}
$$

with the eigenvalue redefined as $\lambda=\lambda_{0,1}$, the time constant redefined as $\tau=\tau_{0,1}$, and the decay coefficient redefined as $\Gamma=\Gamma_{0,1}=2 N_{0}[\sin (\lambda)-\lambda \cos (\lambda)] /[\lambda-\sin (\lambda) \cos (\lambda)]$. The characteristic equation can be simplified to tan $\lambda / \lambda=$ $(1-S a / D)^{-1}$. The eigenvalue, $\lambda$, in this characteristic equation must be solved numerically, given its transcendental form. The solutions for $\lambda$ are then applied to Eq. (5) to find the free-carrier density distribution, $N(r, t)$.

One last simplification can be made by assuming that the nanoparticle is sufficiently small—such that the diffusion length is much larger than the nanoparticle's radius, i.e., $D \gg S_{\mathrm{v}} a$. This approximation produces eigenvalues that approach zero as $D$ approaches infinity. Using Taylor Series expansions and $\lambda \rightarrow 0$, the characteristic equation is simplified to $D \lambda^{2} \approx 3 S_{\mathrm{v}} a$, and Eq. (4) can be generalized to be

$$
\frac{1}{\tau} \approx \frac{1}{\tau_{\mathrm{b}}}+S_{\mathrm{v}} R
$$

where the surface-to-volume ratio is $R=3 / a$ for a sphere. This generalized result is seen in the literature ${ }^{[22]}$. For this case, with small $\lambda$, the decay coefficient is $\Gamma \approx N_{0}$ and the cardinal sine term in Eq. (5) is $\sin (\lambda r / a) /(\lambda r / a) \approx 1$. The free-carrier density distribution is no longer a function of $r$, as it becomes an average free-carrier density given by

$$
N(t) \approx N_{0} \mathrm{e}^{-t / \tau}
$$

When tested, this solution matches the solution from Eqs. (4) and (5) with large values of $D$. The solutions from Eqs. (4) and (5) match the solution from Eq. (2) when computed for a variety of parameters, within $1 \%$ error.

Figure 2(a) simulates two cases for diffusion using Eq. (2). The top half of Figure 2(a) is for a diffusion length on the scale of the radius, such that $D \approx S_{\mathrm{v}} a$, and the bottom half of the figure is for a sufficiently small nanoparticle, such that $D \gg S_{\mathrm{v}} a$. For the first case, with $D \approx S_{\mathrm{v}} a$, the diffusion of free-carriers to the nanoparticle's surface is slow. This yields a nonuniform colourmap, depicting $N(r, t)$, with a higher density near the middle of the nanoparticle in all three of the time steps, $t=0.5 \mathrm{ps}, t=1.0 \mathrm{ps}$, and $t=2.0 \mathrm{ps}$. For the second case, with $D \gg S_{\mathrm{v}} a$, the diffusion is sufficient for rapid transport of free-carriers from the nanoparticle's interior to the surface. This is seen as a uniform colourmap, depicting $N(r, t)$, in all three of the time steps, $t=0.5 \mathrm{ps}, t=1.0 \mathrm{ps}$, and $t=2.0 \mathrm{ps}$. In this latter case, surface recombination becomes the limiting factor defining the free-carrier lifetime.

Figure 2(b) simulates the free-carrier lifetime (being equivalent to the switching time) using Eq. (2). The time constant, $\tau$, is shown versus $a$ and $S_{\mathrm{v}}$ for the case of $D \gg S_{\mathrm{v}} a$, with a long bulk lifetime, $\tau_{\mathrm{b}} \rightarrow \infty$. From the surface plot, $\tau$ is seen to be linear with $a$ and $1 / S_{\mathrm{v}}$, agreeing with the approximation in Eq. (5). The red surface in Figure 2(b) demarcates the region for which $a$ and $S_{\mathrm{v}}$ yield femtosecond (subpicosecond) switching times. Based on the bounds of the red surface, the composition of the semiconductor nanoparticles must have a nanoparticle radius less than $a=30 \mathrm{~nm}$, and a surface recombination velocity greater than $S_{\mathrm{v}}=2000 \mathrm{~m} / \mathrm{s}$. This study proceeds using these conclusions as guides for material selection. 
a
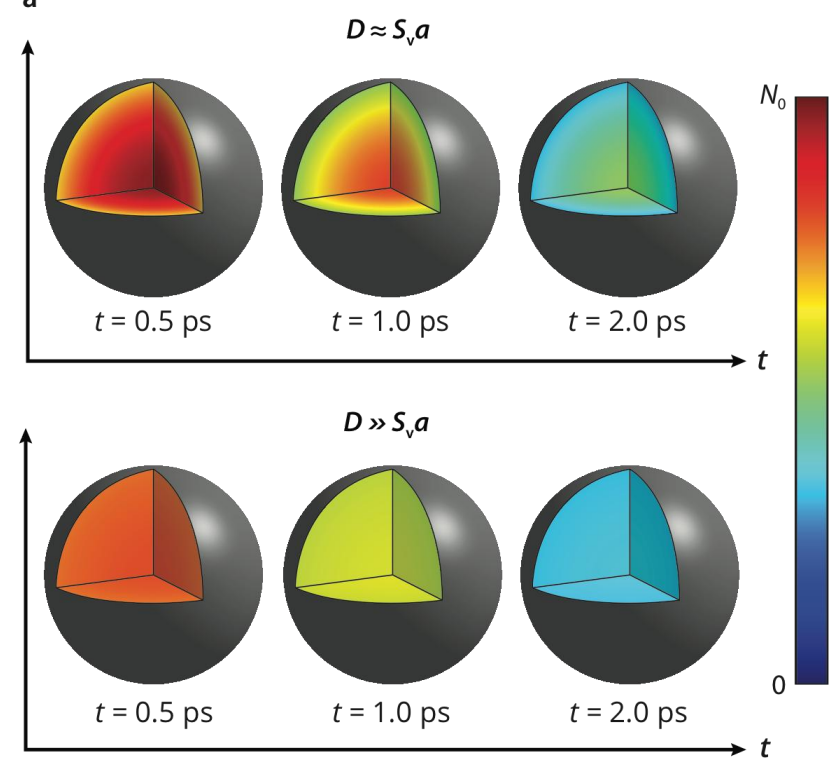

b

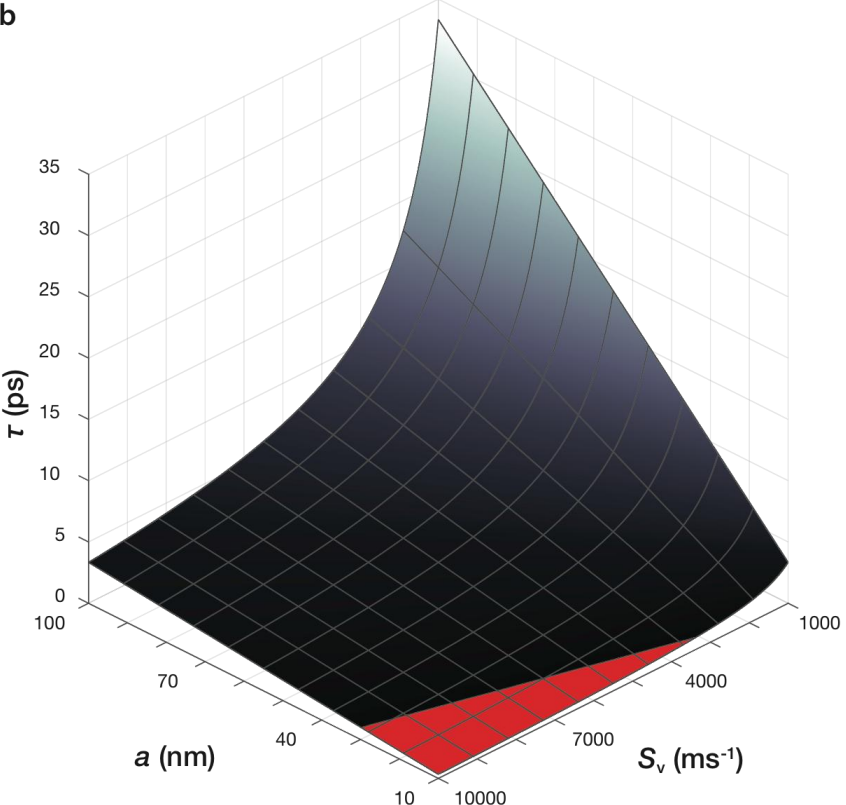

Figure 2. Free-carrier dynamics of semiconductor nanoparticles. (a) The first free-carrier density distribution is given for a small diffusion coefficient relative to the nanoparticle's size, i.e., $D \approx S_{\mathrm{v}} a$. This results in a nonuniform distribution for the time instances of $0.5 \mathrm{ps}, 1 \mathrm{ps}$, and $2 \mathrm{ps}$. The second free-carrier density distribution is given for a sufficiently large diffusion coefficient relative to the nanoparticle's size, i.e., $D \gg S_{\mathrm{v}} a$. This results in a uniform distribution for the time instances of $0.5 \mathrm{ps}, 1 \mathrm{ps}$, and 2 ps. (b) For the case of $D \gg S_{\mathrm{v}} a$ and for a long bulk lifetime, $\tau_{\mathrm{b}}$, the time constant, $\tau$, is plotted as a function of the radius, $a$, and surface recombination velocity, $S_{\mathrm{v}}$. The red region denotes femtosecond (subpicosecond) time constants, which is the desired regime of operation.

\section{EXPERIMENTAL RESULTS}

A series of experiments is conducted with various semiconductor materials to test the proposed AOS in terms of switching energy and switching time. Microspheres made of N-LASF9 glass, with $n=1.83$ and $d=40 \mu \mathrm{m}$, have semiconductor nanoparticles adhered to their surfaces through a dry-coating process ${ }^{[23]}$.

The semiconductor nanoparticle's composition must be chosen such that its bandgap lies between the signal wavelength of $1550 \mathrm{~nm}(0.8 \mathrm{eV})$ and the control wavelength of $775 \mathrm{~nm}(1.6 \mathrm{eV})$. The signal beam is chosen to be 1550 $\mathrm{nm}$, for low losses, integration with contemporary telecommunications systems, and enhanced free-carrier dispersion (scaling with the wavelength-squared ${ }^{[24]}$ ). The control beam is chosen to be $775 \mathrm{~nm}$ to omit the need for a secondary laser source, in lieu of a second harmonic crystal for conversion. The semiconductors, $\mathrm{Si}(1.11 \mathrm{eV}$ indirect bandgap), CdTe (1.56 eV direct bandgap), InP (1.27 eV direct bandgap), and p-type $\mathrm{CuO}\left(1.21 \mathrm{eV}\right.$ indirect bandgap $\left.{ }^{[25]}\right)$ have bandgaps within this desired range and are used in this study. A 775-1550 nm pump-probe differential transmission system is used for testing, with a laser having $90 \mathrm{MHz}$ repetition rate and a $100 \mathrm{fs}$ pulse duration. Such testing reveals the AOS's impulse response. The control (pump) beam is delayed using a translational stage with respect to signal (probe) beams for time-resolved measurements. A lock-in amplifier (with a $100 \mathrm{~ms}$ time constant) is used to measure the signal beam's differential transmission, $\Delta T(t) / T$.

Figure 3(a), (b), (c), and (d) show the all-optical switching results for N-LASF9 glass microspheres with adhered nanoparticles of $\mathrm{Si}, \mathrm{CdTe}, \mathrm{InP}$, and $\mathrm{CuO}$, respectively. Estimates for the minimum switching energies are acquired by comparing the signal strength of each experiment with the beam powers held constant, and are defined for a signal-tonoise ratio equal to one. Estimates for the switching time are acquired by curve-fitting each $\Delta T(t) / T$ response. As the nanoparticles have a large standard deviation, many measurements of each semiconductor are taken. The results shown are the fastest of these measurements, corresponding with the smallest nanoparticles.

The inset in Figure 3(a) shows nanoparticles of Si with a radius of approximately $40 \mathrm{~nm}$. A large switching energy of $1 \mathrm{pJ}$ is estimated, which is due to the poor quantum efficiency of Si from its indirect bandgap. A long switching time of $1.8 \mathrm{ps}$ is measured, which is due to the nanoparticle's large radius. The free-carrier dynamical model, derived in the last section, estimates the surface recombination velocity to be $S_{\mathrm{v}}=7400 \mathrm{~m} / \mathrm{s}$. Based on the results from Figure 2(b), 
this $S_{\mathrm{v}}$ makes it possible for Si to have a femtosecond switching time with a smaller nanoparticle radius. However, such an attempt would not address the high switching energy.

The inset in Figure 3(b) shows nanoparticles of CdTe with a radius of approximately $30 \mathrm{~nm}$. A lower switching energy of $500 \mathrm{fJ}$ is estimated, which is credited to the high quantum efficiency of CdTe from its direct bandgap. A long switching time of $2.3 \mathrm{ps}$ is measured, however, which is due in part from the large nanoparticle radius and in part from the surface recombination velocity. The free-carrier dynamical model estimates CdTe to have $S_{\mathrm{v}}=4300 \mathrm{~m} / \mathrm{s}$. Based on the results from Figure 2(b), this $S_{\mathrm{v}}$ makes it possible for CdTe to have a femtosecond switching time with a smaller nanoparticle radius. However, it is predicted that the radius must be reduced to be below $13 \mathrm{~nm}$. This encroaches upon the Bohr radius of $7.3 \mathrm{~nm}$ for CdTe, which would increase the bandgap past the $1.6 \mathrm{eV}$ control beam photon energy ${ }^{[26]}$.

The inset in Figure 3(c) shows nanoparticles of InP with a radius of approximately $20 \mathrm{~nm}$. A low switching energy of $400 \mathrm{fJ}$ is estimated, which is credited to the high quantum efficiency of InP from its direct bandgap. A short switching time of $900 \mathrm{fs}$ is measured, which is credited to the small nanoparticle radius and high surface recombination velocity. The free-carrier dynamical model, derived in the last section, estimates InP to have $S_{\mathrm{v}}=7400 \mathrm{~m} / \mathrm{s}$. Based on the results, InP meets the demand for femtojoule switching energies and femtosecond switching times.

The inset in Figure 3(d) shows nanoparticles of $\mathrm{CuO}$ with a radius of approximately $20 \mathrm{~nm}$. A low switching energy of $300 \mathrm{fJ}$ is estimated, and an especially short switching time of $350 \mathrm{fs}$ is measured. This switching time is composed of two time constants- that result from free-carrier dispersion as well as state-filling. It is proposed that the p-type lattice forms mid-gap defect states in $\mathrm{CuO}$ nanoparticles. These trapping sites allow for the signal beam to undergo absorption, for transitions of inducing electrons and holes into the mid-gap and valence band, respectively. The arrival of the control beam increases the hole occupancy in the valence band, leading to state-filling and diminished signal beam absorption $^{[18],[19]}$. This effect, combined with a free-carrier absorption contribution, is seen as rapid increase in the transmission. The free-carriers then relax within the bandstructure over a $350 \mathrm{fs}$ time constant, corresponding with a rapid decrease in transmission. This $350 \mathrm{fs}$ time constant is in close agreement with the $400 \mathrm{fs}$ time constant measured for this process in $\mathrm{CuO}$ nanowires ${ }^{[18]}$. Trap-assisted recombination ${ }^{[27]}$ of the relaxed free-carriers then occurs over the next $2.0 \mathrm{ps}$, which is visible in the $\Delta T(t) / T$ response. However, this free-carrier dispersion response a weak $(8 \%)$ contribution the signal amplitude. The $100 \mathrm{fs}$ pulse durations of the control and signal beam are convolved with the (curve-fit) impulse response to produce the quoted two time constants, with an R-squared of 0.9997. It is noted that CuO's 350 fs time constant is found to be roughly independent of the nanoparticle size. This supports the interpretations made here, in attributing this ultrafast response to transitions into mid-gap defect states. Based on the results given here, $\mathrm{CuO}$ meets the demand for femtojoule switching energies and femtosecond switching times. 

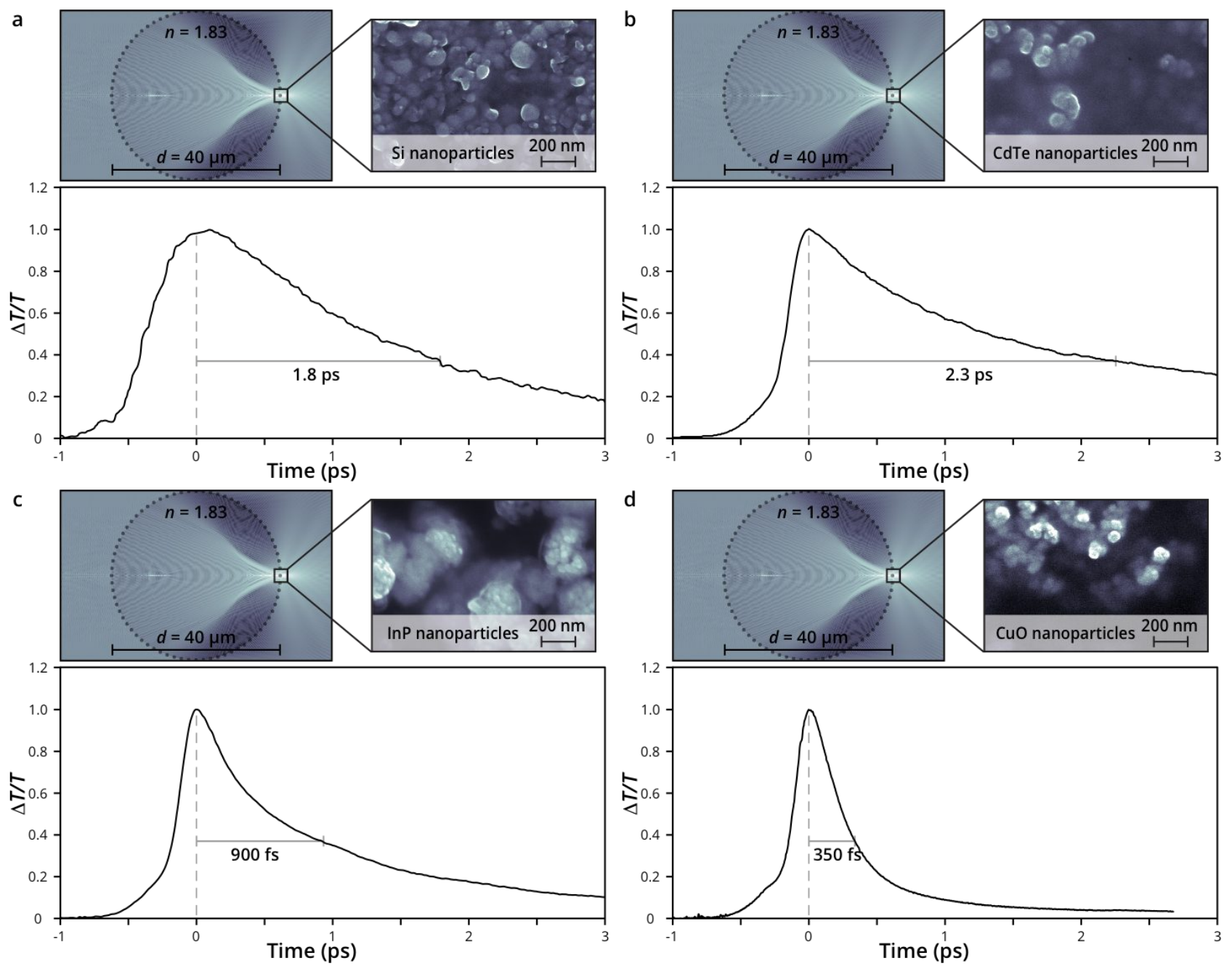

Figure 3. The proposed AOS's impulse responses for varying semiconductor nanoparticles. A photonic nanojet, generated by a microsphere with $d=40 \mu \mathrm{m}$ and $n=1.83$, is formed in the nanoparticle coatings on the microsphere surfaces. The signal beam's differential transmission, $\Delta T / T$, is shown for nanoparticles of (a) $\mathrm{Si}$, (b) $\mathrm{CdTe}$, (c) $\mathrm{InP}$, and (d) $\mathrm{CuO}$, where the minimum switching energies are estimated to be 1 pJ, $500 \mathrm{fJ}, 400 \mathrm{fJ}$, and $300 \mathrm{fJ}$. The switching times are $2 \mathrm{ps}, 2.3 \mathrm{ps,} 900 \mathrm{fs}$, and $350 \mathrm{fs}$, respectively. The figure insets show Lorenz-Mie Theory simulations (with logarithmic intensities) and SEM images of the respective nanoparticles.

\section{CONCLUSION}

In summary, an AOS employing subdiffractional photonic nanojets and select semiconductors nanoparticles was investigated. Femtojoule switching energies were achieved using a superlens, in the form of a dielectric microspheres, with a high-intensity photonic nanojet at the microsphere's rear surface. Simulations with Lorenz-Mie Theory revealed the complex relationship between the microsphere's refractive index and diameter for establishing the photonic nanojet. Using these results, a N-LASF9 glass microsphere with a refractive index of $n=1.83$ and a diameter of $d=40 \mu \mathrm{m}$ was selected. Femtosecond switching times were achieved by using the appropriate semiconductor nanoparticles to coat the microsphere's rear surface. To describe the nonlinear properties of the semiconductor nanoparticles, a free-carrier dynamical model was created. The semiconductor nanoparticles $\mathrm{Si}, \mathrm{CdTe}, \mathrm{InP}$, and $\mathrm{CuO}$ were tested. The minimum switching energies were estimated to be $1 \mathrm{pJ}, 500 \mathrm{fJ}, 400 \mathrm{fJ}$, and $300 \mathrm{fJ}$, and the switching times were measured to be 2 ps, 2.3 ps, $900 \mathrm{fs}$, and 350 fs for the $\mathrm{Si}$, CdTe, $\mathrm{InP}$, and $\mathrm{CuO}$ nanoparticles, respectively. This suggests that $\mathrm{CuO}$ has the optimal AOS characteristics. The proposed AOS meets the demands of emerging fibre optic front-end systems. Future applications, would entail developing the AOS for cascaded on-chip applications, by way of daisy-chained spheres $^{[28]}$, which is typically challenging for all-optical switching ${ }^{[29]}$. The proposed AOS's monolithic architecture and compact footprint are well-suited to this application. Thus, the proposed AOS may become a fundamental element in future all-optical systems. 


\section{REFERENCES}

[1] Miller, D. A. "Device requirements for optical interconnects to silicon chips," Proc. IEEE 97, 1166-1185 (2009).

[2] Husko, C. et al. "Ultrafast all-optical modulation in GaAs photonic crystal cavities," Appl. Phys. Lett. 94, 021111 (2009).

[3] Volz, T. et al. "Ultrafast all-optical switching by single photons," Nature Photon. 6, 605-609 (2012).

[4] Hu, X., Jiang, P., Ding, C., Yang, H. \& Gong, Q. "Picosecond and low-power all-optical switching based on an organic photonic-bandgap microcavity," Nature Photon. 2, 185-189 (2008).

[5] Heuck, M., Kristensen, P. \& Mørk, J. "Energy-bandwidth trade-off in all-optical photonic crystal microcavity switches," Opt. Express 19, 18410-18422 (2011).

[6] Almeida, V. R., Barrios, C. A., Panepucci, R. R. \& Lipson, M. "All-optical control of light on a silicon chip," Nature 431, 1081-1084 (2004).

[7] Nozaki, T. et al. "Sub-femtojoule all-optical switching using a photonic-crystal nanocavity," Nature Photon. 4, 477-483 (2010).

[8] Born, B., Krupa, J. D. A., Geoffroy-Gagnon, S., \& Holzman, J. F. "Integration of photonic nanojets and semiconductor nanoparticles for enhanced all-optical switching," Nat. Comm., 6, 8097 (2015).

[9] Chen, Z. G., Taflove, A. \& Backman, V. "Photonic nanojet enhancement of backscattering of light by nanoparticles: a potential novel visible-light ultramicroscopy technique," Opt. Express 12, 1214-1220 (2004).

[10] Li, X., Chen, Z., Taflove, A. \& Backman, V. "Optical analysis of nanoparticles via enhanced backscattering facilitated by 3-D photonic nanojets," Opt. Express 13, 526-533 (2005).

[11] Lecler, S., et al. "Properties of a three-dimensional photonic jet," Opt. Lett. 30, 2641-2643 (2005).

[12] Born, B., Krupa, J. D. A., Geoffroy-Gagnon, S., and Holzman, J. F. "Ultrafast all-optical switching with photonic nanojets and semiconductor nanoparticles," SPIE Photonics West, 9746-41 (2016).

[13] Geints, Y., Zemlyanov, A. \& Panina, E. "Photonic jets from resonantly excited transparent dielectric microspheres," J. Opt. Soc. Am. B. 29, 758-762 (2012).

[14] Vo, T. D. et al. "Photonic chip based transmitter optimization and receiver demultiplexing of a $1.28 \mathrm{Tbit} / \mathrm{s}$ OTDM Signal," Opt. Express 18, 17252-17261 (2010).

[15] Holzman, J. F. et al. "Ultrafast carrier dynamics in InP photonic crystals," Nanotechnol. 16, 949-952 (2005).

[16] Collier, C. M., Born, B. \& Holzman, J. F. "Ultrafast response of SiC and Si nanocomposite material systems," Electron. Lett. 48, 1618-1619 (2012).

[17] Hamerly, R., \& Mabuchi, H. "Quantum noise of free-carrier dispersion in semiconductor optical cavities," Phys. Rev. A 92, 023819 (2015).

[18] Othonos, A. \& Zervos, M. "Ultrafast hole carrier relaxation dynamics in p-type CuO nanowires," Nanoscale Res. Lett. 6, 622-626 (2011).

[19] Othonos, A., Zervos, M. \& Tsokkou, D. "Tin oxide nanowires: the influence of trap states on ultrafast carrier relaxation," Nanoscale Res. Lett. 4, 828-833 (2009).

[20] Gumstrup, E. M. et al. "Ultrafast carrier dynamics of silicon nanowire ensembles: the impact of geometrical heterogeneity on charge carrier lifetime," J. Phys. Chem. C 118, 8626 (2014).

[21] Gharghi, M., Cheng, C. \& Sivoththaman, S. "Minority carrier recombination in spherical silicon crystals: measurement and process-induced effects," Semicond. Sci. Technol. 23, 125035 (2008).

[22] Jurienas, S., Stepankevieius, V., Strumskis, M. \& Zukauskas, A. "Carrier recombination in CdS nanocrystals under single-electron and high-density excitation," Semicond. Sci. Technol. 10, 301-309 (2008).

[23] Borderieux, S., Wu, C. Y., Bonzongo, J.-C. \& Powers, K. "Control of elemental mercury vapor in combustion systems using Fe2O3 nanoparticles," Aerosol Air Qual. Res. 4, 74-90 (2004).

[24] Collier, C. M., Born, B., Bethune-Waddell, M., Jin, X. \& Holzman, J. F. "Ultrafast photoexcitation and transient mobility of GaP for photoconductive terahertz emission," IEEE J. Quant. Electron. 49, 691-696 (2013).

[25] Zoolfakar, et al. "Nanostructured copper oxide semiconductors: a perspective on materials, synthesis methods and applications," J. Phys. Chem. C 2, 5247-5270 (2014).

[26] Groeneveld, E., Delerue, C., Allan, G., Niquet, Y. M. \& Donega, C. D. "Size dependence of the exciton transitions in colloidal CdTe quantum dots," J. Phys. Chem. C 116, 23160 (2012).

[27] Linnros, J. "Carrier lifetime measurements using free carrier absorption transients. I. Principle and injection dependence," J. Appl. Phys. 84, 275-283 (1998).

[28] Kapitonov, A. M. \& Astratov, V. N. "Observation of nanojet-induced modes with small propagation losses in chains of coupled spherical cavities," Opt. Lett. 32, 409-411 (2007).

[29] Ballarini, D. et al. "All-optical polariton transistor," Nat. Commun. 4, 1778 (2013). 